\title{
EchoGéo
}

27 | 2014

Structures et armatures urbaines

\section{Une « banlieue créative » dans le Grand Paris?}

\section{Boris Lebeau}

\section{(2) OpenEdition}

Journals

Édition électronique

URL : https://journals.openedition.org/echogeo/13718

DOI : 10.4000/echogeo.13718

ISSN : 1963-1197

\section{Éditeur}

Pôle de recherche pour l'organisation et la diffusion de l'information géographique (CNRS UMR 8586)

\section{Référence électronique}

Boris Lebeau, « Une «banlieue créative » dans le Grand Paris ? », EchoGéo [En ligne], 27 | 2014, mis en ligne le 21 janvier 2014, consulté le 10 août 2021. URL : http://journals.openedition.org/echogeo/ 13718 ; DOI : https://doi.org/10.4000/echogeo.13718

Ce document a été généré automatiquement le 10 août 2021.

EchoGéo est mis à disposition selon les termes de la licence Creative Commons Attribution - Pas d'Utilisation Commerciale - Pas de Modification 4.0 International (CC BY-NC-ND) 


\title{
Une « banlieue créative » dans le Grand Paris?
}

\author{
Boris Lebeau
}

\section{Introduction}

1 En raison des mutations du capitalisme moderne qui rapprochent économie et culture (Scott, Leriche, 2005) et du succès retentissant des théories sur la ville créative de $R$. Florida, les projets urbains visant à inscrire les villes dans la modernité de l'économie culturelle se sont multipliés ces dernières années. Le renouveau de vieilles cités industrielles telles Bilbao, Glasgow ou Lille s'est en effet appuyé en grande partie sur des politiques mettant en scène la culture sous ses aspects les plus divers. La circulation rapide de ces expériences dans le cadre d'une sorte de «benchmark urbain » à l'échelle mondiale (Vivant, 2013) permet aujourd'hui l'essor de tels projets dans des contextes variés et parfois atypiques comme nous proposons de le voir avec le cas nord parisien.

2 Atypique, le projet de «territoire de la culture et de la création » nord-parisien l'est tout d'abord en raison du contexte institutionnel dans lequel il se déploie : celui du Grand Paris. Porté par Plaine Commune, communauté d'agglomération de neuf communes (Aubervilliers, Epinay, l'île Saint-Denis, La Courneuve, Pierrefitte, SaintDenis, Saint-Ouen, Stains, Villetaneuse) et 400000 habitants, le projet ne concerne qu'une petite portion de l'agglomération parisienne et non l'ensemble de la métropole. Ensuite, et c'est là sans doute sa plus grande originalité, le projet nord parisien n'a pas pour ambition d'attirer les «manipulateurs de symboles $»^{1}$ selon la formule de R. Reich. Il est destiné au contraire à valoriser les habitants de l'une des banlieues les plus pauvres de la capitale en les présentant comme des créatifs à part entière. Les élus tentent en effet à travers ce projet un pari audacieux qui consiste à présenter les spécificités sociales de cette banlieue (multiculturalisme, marginalité, cultures urbaines) comme une source de créativité dont n'auraient qu'à s'emparer les « industries créatives $»^{2}$ pour enrichir leurs activités et offrir en retour des emplois aux habitants. 
3 Cette déclinaison tout à fait atypique de la «ville créative " pose d'innombrables questions qui relèvent d'ailleurs de divers champs de la géographie (économique, urbaine, sociale, culturelle). Si nous avons choisi de ne privilégier que les aspects politiques, socioculturels et urbanistiques du projet, c'est qu'il nous a semblé impossible d'embrasser l'ensemble des questionnements que celui-ci soulève dans le cadre de cet article.

4 Ce travail repose pour l'essentiel sur une recherche menée depuis deux ans au sein de la MSH Paris-nord. Elle s'appuie sur de nombreux entretiens réalisés à la fois auprès des acteurs institutionnels du Grand Paris (élus, administrateurs publics, ...), et des acteurs économiques et de la culture du territoire (studios de cinéma, réalisateurs, directions de la culture des municipalités, syndicats professionnels, pôles de compétitivité, CCIP, ...). Il mobilise aussi toute une série d'analyses qui ont été menées depuis une dizaine d'années sur les recompositions politiques et socio-économiques de cette périphérie (B. Lebeau, P. Subra, H. Vieillard-Baron...).

5 Nous verrons donc comment ce projet de "territoire de la culture et de la création » a émergé dans le contexte politique particulièrement complexe du Grand Paris. Nous montrerons ensuite qu'il relève plus de la spéculation que d'un fonctionnement socioéconomique solidement ancré dans le territoire. Enfin, nous analyserons quels sont les freins à sa mise en œuvre sur le plan de l'aménagement et de sa gouvernance.

6 Ainsi, nous tenterons de montrer que ce projet qui marque une nouvelle étape dans le processus de réintégration économique et fonctionnelle de cette périphérie, n'est probablement pas de nature à pallier ses difficultés sociales.

\section{Un projet technocratique dans le cadre du Grand Paris}

7 Visant à positionner Paris sur des secteurs d'activités à fort pouvoir symbolique et de croissance, le projet de «territoire de la culture et de la création » est un des éléments de la politique de compétitivité territoriale impulsée en 2008 par le gouvernement. Piloté depuis plus de deux ans par les services de Plaine Commune, le projet de cluster est aussi au cœur des enjeux d'autonomie des pouvoirs locaux dans le cadre du Grand Paris. L'appréhender nécessite d'exposer au préalable la complexité des jeux politiques internes à la métropole parisienne.

\section{Un projet à vocation internationale dans le cadre des réflexions sur le Grand Paris}

8 Initié en 2007 par le Président Sarkozy (Subra, 2012), le Grand Paris est imaginé pour résoudre les problèmes de transport, de gouvernance, de compétitivité économique et de logement que connaît la capitale. Outre les missions d'expertise confiées à E. Balladur pour la gouvernance et aux dix équipes d'architectes pour la prospective et la communication internationale, l'État se dote d'un Secrétariat d'Etat éponyme qu'il confie à $\mathrm{C}$. Blanc pour définir concrètement le projet d'aménagement métropolitain. Ce dernier imagine alors un dispositif territorial qui consiste à relier par un métro les pôles économiques situés à la périphérie de Paris (La Défense et ses fonctions d'encadrement économiques et financières, Saclay et ses activités de recherche, le Bourget et les activités aéronautiques...) pour y renforcer l'activité et construire des 
logements. A ce jeu d'affichage des spécialisations économiques et fonctionnelles, la communauté d'agglomération Plaine Commune se voit attribuer le rôle de « cluster de la création » en lien avec la présence d'activités audiovisuelles et cinématographiques. Or, cette labellisation n'est pas anodine. Si elle renvoie à une certaine spécialisation économique du territoire, qui concentre, il est vrai, une part importante des plateaux de tournage de cinéma ( $50 \%$ du total national) et de l'audiovisuel (près de $80 \%$ des émissions de télévision sont tournées à la Plaine Saint-Denis), ces activités ne représentent toutefois qu'une faible part de l'emploi local (2,4\%). Le choix de l'État répond alors à divers impératifs. Le premier est d'accompagner l'ambitieux projet de Cité du cinéma lancé à Saint-Denis par le réalisateur L. Besson. Ce " temple du cinéma » qui regroupe dans une ancienne centrale électrique en bord de Seine toutes les étapes de la fabrication d'un film (studios de tournage, studios de post-production, salles de projection, bureaux pour les sociétés de production) vise à créer un lieu capable de rivaliser avec les plus grands centres de production cinématographiques mondiaux comme Hollywood (Los Angeles), Pinwood (Londres), ou Babelsberg (Berlin). Or, il se trouve que depuis 2008, l'État est partie prenante de ce projet par l'intermédiaire de la Caisse des dépôts et consignations qui acquiert pour 150 millions d'euros le foncier de l'opération ${ }^{3}$.

9 Le second impératif était la création à la Plaine Saint-Denis ${ }^{4}$ d'un pôle d'interconnexion des transports qui offrirait une liaison directe entre La Défense et l'aéroport Charles de Gaulle. Liaison à laquelle le Président, ancien élu des Hauts-de-Seine, semblait particulièrement attaché. Enfin, cette « labellisation », qui donne une image positive du territoire, à contre-pied des clichés stigmatisant sur la banlieue, apparaissait aussi comme un moyen de s'assurer le soutien des élus de ce bastion communiste.

C'est dans ce contexte qu'en 2008, C. Blanc propose à P. Braouezec (Président de Plaine Commune) de constituer un établissement public pour construire de toute pièce un « cluster de la création » à la Plaine Saint-Denis.

\section{L'appropriation locale du projet}

11 L'accueil réservé par les élus locaux au projet est ambigu. Séduits d'être présentés comme les « créatifs » du Grand Paris, ils sont aussi extrêmement méfiants à l'égard de l'État dont les velléités de reprise en main de l'aménagement francilien sont réelles comme le laissent alors présager les premières conclusions du rapport Balladur qui préconise la fusion des trois départements de la petite couronne. P. Braouezec fonde alors avec le Maire de Paris et d'autres élus de gauche la " conférence métropolitaine » (Subra, 2012), dont l'objectif est de faire pression sur le gouvernement pour qu'il abandonne son projet de gouvernance. Transformée depuis en «Syndicat Paris Métropole », cette structure de coopération, que P. Brouezec préside en 2012, regroupe aujourd'hui 192 adhérents (communes, groupements de communes, départements, Région, établissements publics) de toutes tendances politiques. On l'aura compris, l'implication des élus dans le projet a beaucoup à voir avec la volonté de conserver une autonomie de décision au niveau local.

Mais, dans le même temps, les élus vont faire évoluer le projet gouvernemental dans un sens plus favorable à l'agglomération. Ils obtiennent alors deux concessions majeures. La première concerne l'abandon de l'établissement public en faveur d'une contractualisation entre l'État et Plaine Commune. Ce dispositif du contrat de 
développement territorial (CDT) sera d'ailleurs repris et généralisé pour l'ensemble des intercommunalités franciliennes concernées par le projet de transport du Grand Paris.

La seconde concession concerne l'élargissement du contenu du projet et de son périmètre. Les élus estiment en effet que le « cluster de la création » a une connotation à la fois trop économique et trop technocratique. En outre, il valorise Paris comme capitale du cinéma, sans se soucier des problèmes de Plaine Commune (chômage, exclusion, violence, ...). Les élus ont donc à cœur d'imaginer un véritable projet intercommunal qui dépasse le cadre du quartier de la Plaine Saint-Denis et qui profite à l'ensemble des habitants du territoire. Dans cette perspective, ils vont progressivement intégrer de nouvelles composantes dans le projet.

Ils estiment tout d'abord que le potentiel créatif du territoire ne saurait se limiter aux industries du cinéma et de l'audiovisuel. L'agglomération est en effet riche d'initiatives et d'institutions culturelles diverses (deux scènes nationales, le festival de musique classique et la Basilique de Saint-Denis, les Archives nationales, le marché aux puces de Saint-Ouen, l'académie du cirque Fratellini, Zingaro, ses conservatoires de musiques dont deux d'envergure régionale, son réseau de médiathèques...) et qu'à ce titre, c'est l'ensemble de la filière culturelle publique qui doit intégrer le projet. Ils font valoir également la présence sur le territoire d'un ensemble d'activités créatives issues du numérique comme Orange business service, le siège social de SFR ou encore les datacenters ${ }^{5}$, en lien avec l'exceptionnelle densité du réseau de fibres optiques traversant la communauté d'agglomération. Enfin, les élus souhaitent placer la population au cœur du projet. Dans cette perspective, le territoire social est désormais présenté comme un milieu créatif susceptible d'attirer et d'alimenter en idées et en main-d'œuvre tout un ensemble d'activités culturelles et créatives pour partie déjà implantées sur le territoire (audiovisuel, numérique, arts vivants...) ou qu'il conviendrait d'attirer grâce à des aménagements et des initiatives appropriés.

15 Le projet de "cluster de la création " proposé par l'État, qui était d'ailleurs plus un cluster autour des métiers de l'image, devient sous la houlette de Plaine Commune un projet de territoire créatif, désigné depuis 2011 «territoire de la culture et de la création ».

\section{La banlieue comme milieu créatif}

16 Le concept de ville créative, qui a fait l'objet de nombreuses critiques tant sur la plan idéologique que méthodologique (Jouve, 2007 ; Keil et Boudreau, 2010 ; Bourdain, 2010 ; Vivant, 2011) semble a priori peu approprié à cette périphérie sociale. L'idéologie libérale qu'il véhicule tout comme la gentrification qu'il appelle semblent à la fois peu conforme aux idées politiques des édiles et peu en phase avec les intérêts de la population locale. Loin de se résumer pourtant à son acception traditionnelle, nous verrons que la notion de ville créative est ici totalement adaptée aux spécificités sociales de ce territoire.

\section{De la ville créative à la « banlieue créative »}

17 Le concept de ville créative a quelque chose de profondément élitiste. Il suggère en effet de fonder le développement urbain sur la concentration des activités créatives et sur la captation d'une population d'artistes, de créateurs, de jeunes entrepreneurs, de 
chercheurs, ... issue des catégories socioprofessionnelles supérieures, appelées par son concepteur (R. Florida) "classe créative». Ce faisant, la notion de ville créative a souvent servi d'habillage conceptuel à de banales politiques de gentrification, notamment en Amérique du Nord (Toronto). Or les élus de Plaine Commune se sont toujours défendus de vouloir exclure les plus démunis de leur territoire ${ }^{6}$.

Par ailleurs, le concept a parfois servi à promouvoir la libéralisation des services urbains, comme ce fut le cas à Toronto dans le contexte d'austérité qui caractérisait le Canada des années 1990. Dans ce cas, la promotion de la ville créative correspondait en quelque sorte à la promotion de la ville entrepreneuriale (Le Galès, 2007). Derrière le discours apparemment progressiste des élus torontois se cachait un autre message en direction de leurs administrés que l'on pourrait résumer ainsi : « soyez entreprenant et créatif, prenez-vous en main car en raison de ses difficultés financières, la municipalité ne pourra plus rien pour vous ».

19 Il peut donc paraître surprenant qu'un tel concept soit aujourd'hui porté par les élus d'une majorité communiste a priori très éloignée de l'idéologie néolibérale et de l'empowerment (entendu comme le développement des capacités d'agir des individus, mais ici surtout de l'esprit entrepreneurial) et qu'il guide désormais la stratégie territoriale de l'agglomération. Séduits par un concept de marketing territorial investi de valeurs positives et consensuelles (culture, création, figure de l'artiste entrepreneur) lequel, comme le développement durable est suffisamment flou et évocateur pour que l'on ne puisse s'y opposer, les élus de Plaine Commune, qui ne semblent pas toujours conscients de la dimension idéologique du concept, ont toutefois une interprétation du territoire créatif qui ne saurait être réduite à celle de Florida et à son acception anglosaxonne de ville entrepreneuriale. Que recouvre donc une telle proposition pour les élus locaux?

En suggérant que la banlieue nord de Paris est un territoire créatif, Plaine Commune tente à l'évidence de transformer les représentations accolées à ce territoire largement stigmatisé il est vrai pour ses problèmes (pauvreté, insécurité, chômage, tensions interculturelles, etc.). En témoigne par exemple la volonté de Plaine Commune de valoriser les cultures urbaines, « longtemps dévalorisées et insuffisamment soutenues » et considérées aujourd'hui par l'agglomération comme une «marque de fabrique » et un "point fort du territoire", tant elles "représentent une nouvelle forme de réussite sociale et artistique et de mobilisation des milieux populaires, à contre-courant de l'image d'échec et de relégation ou de passivité à laquelle sont parfois associés le territoire et les habitants de la Seine-Saint-Denis et la banlieue nord de Paris. » (Contrat de Développement Territorial 2014-2030, p. 90).

21 Ce faisant, les élus suggèrent qu'il existerait sur place un «terreau social » propre à la création qu'on ne retrouverait du reste pas ailleurs dans la métropole. Au-delà de la présence réelle des studios de cinéma et de la richesse de la vie culturelle locale liée aux héritages du socialisme municipal, cette idée de «territoire créatif » semble avoir deux origines dans l'esprit des promoteurs du projet ${ }^{7}$.

22 Elle renvoie tout d'abord à l'idée que la «culture de banlieue » (ou ce que l'on nomme aussi les cultures urbaines) est par essence créative, rejoignant ainsi un présupposé selon lequel, l'innovation qu'elle soit sociétale, économique, voire scientifique naît dans les marges. Largement prégnante dans l'ouest de la Seine-Saint-Denis, cette « culture de banlieue » trouve ici d'illustres représentants parmi lesquels figurent le groupe de rap NTM, le slameur Grand corps malade originaires de Saint-Denis et des danseurs ou 
graffeurs moins connus du grand public. Tous ont, il est vrai, permis d'ériger cette culture en art. Il n'est d'ailleurs pas surprenant qu'en raison de la diversité de ses expressions (musique, danse, graffiti, sport) certains soient tentés de faire un parallèle (même inconscient) avec les industries créatives, composées comme on l'a vu d'une grande variété d'activités ... même si la dimension industrielle de ces formes d'expression reste très limitée voire inexistante. Néanmoins, cette culture est aujourd'hui universelle et il semble difficile de s'arroger un monopole en la matière. Quant à la richesse de la vie culturelle locale, certes florissante, elle reste peu de chose face à l'écrasante supériorité parisienne.

Le territoire créatif est aussi pour les élus locaux l'occasion de valoriser la diversité culturelle qui est souvent présentée comme une "richesse». Sur ce territoire, où les étrangers représentent plus de $30 \%$ de la population et où vivent des ressortissants de 136 nationalités, le melting-pot serait donc source d'innovations sociales et de créativité. La marginalité et la diversité ne seraient donc plus des handicaps, mais des atouts, et pourraient même nourrir les entreprises (du cinéma, de l'animation, du jeu vidéo ...) de nouveaux talents et de nouvelles idées. Ces entreprises seraient donc susceptibles d'offrir en retour des emplois et des occasions d'insertion sociale aux habitants de cette banlieue. Là encore, que des décideurs locaux aient l'intuition que la diversité des cultures en présence soit une source d'enrichissement potentiel dans le cadre d'une économie culturelle et créative n'étonnera guère. D'ailleurs le cinéma ou l'audiovisuel se sont largement nourris ces dernières années de talents venant des banlieues les plus pauvres (Djamel Debouze, Omar Sy, Tahar Rahim ...).

On comprend qu'ainsi pervertie, la notion de «ville créative » n'a plus grand chose à voir avec son acception élitiste et libérale.

Cette idée, au demeurant séduisante, interroge néanmoins sur cette façon singulière qu'ont les élus d'exalter la "culture de banlieue " au nom de la lutte contre ses expressions les plus problématiques et les plus violentes. D'autre part, comme le souligne E. Vivant en parlant des cultures alternatives et de la scène off, vouloir institutionnaliser cette culture de la marginalité (si tant est que cela soit possible) n'est-ce pas la condamner à disparaître ? Enfin, dans l'économie libérale, l'injonction qui est faite aux individus d'être indépendants pénalise et culpabilise ceux qui précisément sont trop démunis pour assurer leur autonomie comme le montre Robert Castel (2009). La rhétorique de la ville créative qui pousse plus loin encore cette logique en invitant les individus à être non seulement indépendants mais créatifs (et demain intelligents ${ }^{8}$ ), risque fort de déstabiliser les populations locales, insuffisamment armées pour répondre à ces injonctions. Il y a manifestement sur ce sujet une attitude ambiguë de la part des élus qui dénote une certaine méconnaissance des concepts qu'ils manipulent.

\section{La banlieue créative à l'épreuve de l'emploi}

Il est a priori difficile de savoir si l'approche développée par les élus est vertueuse ou spécieuse. Il conviendrait peut-être de mesurer à plus long terme l'effet de cette rhétorique sur les représentations que les habitants ont d'eux-mêmes et sur celles que les entreprises ont de la population et du territoire. Mais, pour le moment, l'un des rares éléments tangibles dont nous disposons est de confronter ces ambitions politiques à la réalité de l'emploi. 

secteurs d'activités. Toutefois, en raison de l'important décalage existant entre les qualifications des actifs résidents et des actifs travaillant à Plaine Commune, tout porte à croire que ces activités offrent peu d'emplois aux habitants: $75 \%$ d'entre eux travaillent d'ailleurs hors de l'intercommunalité et inversement $75 \%$ des actifs travaillant à Plaine Commune n'y résident pas.

Tableau 1 - La non concordance entre les emplois offerts et les qualifications des résidents

\begin{tabular}{|l|l|l|}
\hline & Plaine Commune & Ile-de-France \\
\hline Part des actifs résidents ne disposant d'aucun diplôme & $39 \%$ & $17,1 \%$ \\
\hline Part des actifs résidents diplômés du supérieur & $7,2 \%$ & $36,7 \%$ \\
\hline Part des cadres dans la population active résidente & $7,1 \%$ & $28,3 \%$ \\
\hline Part des cadres au lieu de travail & $22,5 \%$ & $28,5 \%$ \\
\hline
\end{tabular}

Source : INSEE, RP 2010.

Dans les métiers créatifs à haut niveau de capital culturel et de formation, tout porte à croire que cette tendance est amplifiée comme l'attestent les statistiques de la filière image.

31 Valorisantes pour le territoire, les activités créatives sont sans doutes celles qui en terme d'emplois sont les plus inaccessibles aux populations locales, car plus encore que des diplômes, elles exigent un capital culturel, social et symbolique (au sens de Bourdieu). Or c'est précisément ce dont manquent les habitants de ce territoire, souvent des primo-arrivants. Ce clivage est un obstacle évident au projet 
intercommunal et tend à montrer que le concept de ville créative n'est peut-être pas le remède miracle aux maux de cette banlieue. En tous cas, ces quelques données sur l'emploi nous rappellent que la créativité comme le territoire ne se décrètent pas.

\section{Une nouvelle étape dans la réintégration économique et fonctionnelle du territoire}

32 Le principal défi qui se pose à l'aménagement est évidemment de créer ex nihilo un environnement urbain propre à la création. Il s'agit là d'un défi d'autant plus conséquent qu'en la matière, les modèles sont rares et que le territoire part avec de lourds handicaps. En outre, les impératifs de l'aménagement francilien mis en œuvre dans le cadre du Grand Paris sont plutôt de nature à renforcer les transformations économiques déjà en cours sur le territoire qu'à les infléchir dans le sens d'un territoire créatif supposé plus profitable aux habitants.

\section{Les défis posés à l'aménagement}

Au-delà des déclarations d'intention, le projet de "territoire de la culture et de la création » doit s'appuyer sur un ensemble de réalisations qui relèvent du champ de l'urbanisme. Il s'agit en effet pour Plaine Commune de proposer une offre immobilière pour les entreprises créatives venant de différents secteurs d'activité, mais aussi des ateliers pour les artistes, ainsi que des lieux d'échange ou de rencontre entre les créatifs, les entreprises et les habitants. Or la chose est sans doute plus facile à imaginer qu'à mettre en œuvre.

Si, à Montreuil, les artistes et les créatifs ont trouvé dans l'entrelacement d'un habitat pavillonnaire et de petites industries diffuses un milieu propice à leur implantation résidentielle et professionnelle, il en va tout autrement à Plaine Commune. Dans le quartier de la Plaine Saint-Denis, les industries gazières, chimiques, métallurgiques ... ont laissé des friches d'une taille considérable que seuls des grands porteurs de projets peuvent aujourd'hui se réapproprier (sièges sociaux, centres de recherche, Cité du cinéma ...), laissant peu de place à la bohème artiste. Par ailleurs, l'environnement y a été «mutilé » par les infrastructures de transport (autoroutes, voies ferrées) provoquant un déficit d'urbanité lourd de conséquences à l'heure où l'agglomération cherche à créer un environnement urbain propice à l'accueil des créatifs. Quant aux grands ensembles d'habitat social, qui représentent plus de $40 \%$ des logements du territoire, ou l'habitat ancien dégradé des centres de Saint-Denis ou d'Aubervilliers (sur)occupé par les populations les plus marginalisées et en proie à de nombreux trafic ${ }^{11}$, ils ne constituent $a$ priori pas un cadre très approprié au développement de ces activités. Enfin, le tissu pavillonnaire de piètre qualité à Pierrefitte, Villetaneuse, Stains ou La Courneuve, ne présente aucun des attributs de la centralité susceptibles d'attirer les entreprises créatives ou les populations d'artistes.

Dans le CDT signé en avril 2013, l'agglomération acte néanmoins son intention de réserver $30 \%$ des nouvelles constructions aux activités créatives. L'agglomération souhaite proposer une offre immobilière adaptée à ces activités en jouant sur la modularité, notamment en proposant des locaux mixtes d'activités et d'habitation, ainsi que des lieux de rencontre. Evidemment l'idée de planifier de toute pièce un 
quartier «bohème " paraîtra utopique tant la chose est habituellement affaire de spontanéité.

Le $6 \mathrm{~B}$, lieu où réside aujourd'hui plus d'une centaine d'artistes à Saint-Denis, est assez emblématique de la difficile planification des espaces créatifs. Cet ancien bâtiment administratif est le seul vestige d'un ancien espace d'activités aujourd'hui en reconversion. Le propriétaire des lieux, redoutant d'être squatté durant la longue phase des travaux, a décidé en 2010 de proposer un bail précaire à des artistes parisiens intéressés par les lieux. Situé à quelques centaines de mètres seulement de la gare de Saint-Denis, et par conséquent à moins d'un quart d'heure du centre de Paris, les quelques 150 résidents (artistes, plasticiens, designers, graphistes, architectes ...) présents peuvent jouir pleinement des $77000 \mathrm{~m}^{2}$ de locaux et des terrains alentours sans crainte d'importuner le voisinage. Saisissant l'opportunité, Plaine Commune a décidé en 2012 d'implanter au 6B ce qu'elle appelle "l'atelier », sorte de «living lab " ou d'espace collaboratif où serait élaboré le projet de territoire créatif avec les habitants, les artistes et les industriels. Cette volonté est pourtant contradictoire avec les aménagements qu'elle a elle-même planifiés. En effet, depuis septembre 2013, la mise en chantier de 550 logements et $25000 \mathrm{~m}^{2}$ de bureaux a débuté sur le secteur, ce qui devrait à court terme mettre fin au bail précaire dont bénéficiaient jusque-là les artistes et provoquer leur départ. A moins que l'agglomération choisisse d'indemniser le promoteur pour assurer leur maintien.

Cet exemple est emblématique des contradictions de ce projet mais aussi des dynamiques immobilières à l'œuvre sur le territoire, et que le Grand Paris va à l'évidence renforcer.

\section{Le Grand Paris : une nouvelle étape dans la fragmentation du territoire}

Le contrat signé avec l'État en avril 2013 oblige Plaine Commune à construire 4200 logements par an en échange de l'arrivée de nouveaux transports. Cet objectif qui impose le doublement du nombre des constructions laisse peu de place à la fantaisie. En raison des nombreuses difficultés inhérentes à la construction en milieu dense (manque de place, prix du foncier, dépollution des sols ...), il n'est pas exclu que pour satisfaire les exigences du CDT, Plaine Commune soit amenée à réviser à la baisse les surfaces qu'elle entend dédier aux activités créatives.

D'autre part, la promesse de nouveaux transports, là où précisément l'agglomération souhaite construire les locaux d'activités créatives, risque de provoquer un emballement du marché immobilier alors que Plaine Commune n’a pas véritablement la maîtrise du foncier sur ces secteurs. D'ailleurs l'intérêt des entreprises pour ce territoire est croissant, comme en témoigne l'arrivée récente de Veolia et de SFR. Avec le Grand Paris, ce sont les dernières friches industrielles (Pleyel, Porte d'Aubervilliers, Docks de Saint-Ouen) qui seront gagnées par la tertiarisation de la Plaine Saint-Denis. Dans ces conditions, les promoteurs immobiliers se montrent peu enthousiastes à l'idée de réserver $30 \%$ des nouvelles constructions aux activités créatives, mettant en avant l'absence de marché sur ce type d'offre et surtout la moindre rentabilité de tels locaux. Il semblerait que les aménagements du Grand Paris soient plutôt de nature à accélérer la tertiarisation de la Plaine Saint-Denis et à renforcer la fragmentation du territoire de Plaine Commune qui apparaît plus que jamais écartelé entre sa réussite économique et 
ses problèmes sociaux. Dans ces conditions, l'ambition de bâtir un territoire pour les créatifs et dans l'intérêt des habitants relève sans doute plus de l'utopie que d'un diagnostic lucide sur la réalité des mécanismes métropolitains.

\section{Conclusion}

41 Le projet de «territoire de la culture et de la création » repose sur un ensemble de conjectures et une rhétorique parfois incantatoire qui lui confère un caractère hautement spéculatif, si bien que la question de savoir quelles sont ses véritables finalités reste ouverte. Est-ce de conserver une autonomie de décision au niveau local dans le projet métropolitain, de négocier des équipements dans le cadre du Grand Paris, de flatter la population en lui témoignant l'estime de ses représentants ou bien est-il tout simplement une fausse bonne idée pour sortir le territoire de ses problèmes? Probablement est-il tout cela à la fois. S'il est incontestablement une réussite politique pour les élus locaux dont l'image sort renforcée à l'approche des élections municipales (même s'ils n'échapperont pas à une intégration de la communauté d'agglomération dans la «Métropole de Paris» en 2016), les choses semblent plus mitigées voire inquiétantes sur le plan du territoire.

L'écart abyssal entre l'engouement politique que suscite ce projet, qui porte l'espoir d'une réintégration fonctionnelle et sociale de ces quartiers dans le cadre d'une économie culturelle et créative, et la réalité des fonctionnements socio-économiques laisse perplexe. Elle conduit en tous cas à penser que ce qui est réellement en jeu dans l'affaire est plutôt d'ordre symbolique. Doit-on en conclure que les élus en sont réduit à manipuler les symboles faute de pouvoir apporter des réponses plus concrètes aux problèmes de ces banlieues?

Enfin, ce projet amène à penser que face à la multiplication de concepts aussi ambigus que ceux de "ville créative » ou de "villes intelligentes ", proposés par des auteurs dont les motivations sont parfois plus idéologiques et commerciales que scientifiques, une véritable pédagogie des élus et des décideurs locaux ne serait certainement pas superflue. Elle éviterait que les mêmes recettes soient appliquées en tout lieu, sans aucun discernement de ce qui fait la spécificité des territoires, et qu'à une certaine convergence des problèmes de la ville dans la mondialisation on ne réponde par la standardisation des solutions politiques.

\section{BIBLIOGRAPHIE}

Boste F, 2011 (coordination). Le territoire français, la désindustrialisation et les délocalisations. $B A G F, \mathrm{n}^{\circ} 2,2011,115 \mathrm{p}$.

Bourdain A, 2005. La classe créative existe-t-elle ? Urbanisme, $\mathrm{n}^{\circ} 344$.

Castel R, 2009. La montée des incertitudes, travail, protections, statut de l'individu. Paris, Points Seuil, $457 \mathrm{p}$. 
CDT Plaine Commune, 2013.

Florida R, 2004. Cities and the créative class. New-York, Routledge, 208 p.

Florida R, 2002. The Rise of the Creative Class. And How it's Transforming Work, Leisure, Community and Everyday Life. New York, Basic Books, 480p.

Grésillon B, 2008. Ville et création artistique : pour une approche de la géographie culturelle. Annales de géographie, $\mathrm{n}^{\circ}$ 660-661, p. 179-198.

IAURIF, 2003. Les industries culturelles en Ile-de-France. 196 p.

Jouve B, 2007. Urban societies and dominant political coalitions in the internationalization of cities. Environment and Planning: Government and Policy, $\mathrm{n}^{\circ} 25$.

Keil R, Boudreau J-A,, 2010. Le concept de ville créative : la création réelle ou imaginaire d'une forme d'action politique dominante. Métropoles, $\mathrm{n}^{\circ}$ 7, $12 \mathrm{p}$.

Lebeau B, 2010. Analyse du recensement de 2006 à Plaine Commune. Saint-Denis au fur et à mesure, $\mathrm{n}^{\circ} 54,1^{\text {er }}$ trimestre 2012.

Lebeau B, 2007. Intégration fonctionnelle et marginalisation sociale en Plaine-de-France. BAGF, n'3-2007, p. 315-326.

Le Galès P, 2003. Le retour des villes européennes : sociétés urbaines, mondialisation, gouvernement et gouvernance. Paris, Presses de Sciences Po, $454 \mathrm{p}$.

Liefooghe C, 2010. Lille 2004, capitale de la culture ou quête d'un nouveau modèle de développement. Méditerranée, $\mathrm{n}^{\circ} 114, \mathrm{p} .34-45$.

Markusen A, 2006. Urban development and the politics of a creative class: evidence from a study of artists. Environment and Planning, $\mathrm{n}^{\circ} 38$.

Pôle média Grand Paris, 2012. Étude sur l'emploi et la formation dans la filière image du nord parisien. $39 \mathrm{p}$.

Reich, R, 1993. L'économie mondialisée. Paris, Dunod, 336 p.

Scott A, Leriche F, 2005. Les ressorts géographiques de l'économie culturelle : du local au mondial. L'espace géographique, $\mathrm{n}^{\circ} 3 / 2005$, tome 34, p. 207-222.

Subra P, 2012. Le Grand Paris : géopolitique d'une ville mondiale. Paris, A. Colin, 307 p.

Subra $P, 2011$. Les municipalités communistes face au défi de la désindustrialisation. BAGF, $\mathrm{n}^{\circ} 2$, p. 174-181.

UNESCO, 2010. Rapport annuel sur l'économie créative. 281 p.

Vieillard-Baron H, 2011. La Plaine Saint-Denis : un ancien territoire industriel. BAGF, $\mathrm{n}^{\circ} 2$, p. 164-173.

Vivant E, 2013. Qu'est-ce que la ville créative? Paris, PUF, 92 p.

\section{NOTES}

1. R. Reich utilise cette formule pour désigner, un peu comme R. Florida, les catégories socioprofessionnelles sur lesquelles repose la mondialisation des échanges (ingénieurs, chercheurs, juristes, financiers, spécialistes de la communication...).

2. Selon l'UNESCO, on regroupe sous le vocable «industries créatives " les activités suivantes: marché de l'art et des antiquités, artisanat d'art, architecture, spectacle vivant, photographie, 
publicité, mode, design et graphisme, édition, audiovisuel, cinéma, jeux vidéo-logiciels et loisirs numériques, musique.

3. D'après Le Monde du 18-11-13, le montage financier aurait été négocié en 2008 par C. Guéant alors secrétaire général de la présidence de la République.

4. La Plaine Saint-Denis recouvre sur 800 ha, à cheval sur Saint-Denis, Saint-Ouen et Aubervilliers, l'essentiel du sud de l'intercommunalité. Ancien espace industriel en reconversion, il est devenu depuis la fin des années 1990 un pôle d'activités majeur en île-de-France (65 000 emplois aujourd'hui).

5. Centre de stockage de données numériques.

6. Bien que le discours puisse ne pas être au diapason des ambitions politiques, l'agglomération envisage tout de même de construire $40 \%$ de logements sociaux d'ici 2020 (SCOT, PLH).

7. Nous employons le conditionnel car après de nombreux entretiens avec les différents protagonistes, ces représentations semblent prégnantes sans pour autant être véritablement formalisées.

8. On se réfère ici aux travaux de R. Giffinger qui identifie 6 critères pour définir une "ville intelligente" (une économie intelligente, une mobilité intelligente, un environnement intelligent, des habitants intelligents, un mode de vie intelligent et, enfin, une administration intelligente).

9. INSEE, RP 2006.

10. Pôle média Grand Paris, 2012. Étude sur l'emploi et la formation dans la filière image du nord parisien. $39 \mathrm{p}$.

11. Trafic de drogue, prostitution et marchands de sommeil ont envahi de nombreux immeubles du centre de Saint-Denis et d'Aubervilliers.

\section{RÉSUMÉS}

Le projet de «territoire de la culture et de la création» porté par la communauté d'agglomération Plaine Commune dans le cadre du Grand Paris constitue une déclinaison tout à fait originale du concept de «ville créative » dans le cadre d'une banlieue pauvre. En assimilant la marginalité sociale à la créativité, ses concepteurs nous interrogent sur la place que pourraient à l'avenir occuper ces quartiers populaires dans le cadre d'une économie culturelle et créative. En démêlant ce qui relève du politique, du marketing territorial et du fonctionnement socioéconomique du territoire, nous tenterons d'examiner quelle peut être la portée d'une telle proposition.

The community of Plaine Commune is implementing a project of a «territory of culture and creation » within the Grand Paris. It is a completely original version of the concept of a creative city, set in a deprived suburb. Indeed, its designers liken social fringe to creativity and in doing so they question the place that these poorer districts could hold within a cultural and creative economy in the future. We'll draw distinctions between what is political, what is territorial marketing, and what is the socio-economic functioning of the territory and we will study what impact such a proposal can have. 
INDEX

Keywords : creative city, suburb, Grand Paris, creative industry, urban planning

Mots-clés : ville créative, banlieue, Grand Paris, industrie créative, aménagement

Thèmes : Sur le Champ - Sur le Terrain

\section{AUTEUR}

\section{BORIS LEBEAU}

Boris Lebeau, borislebeau@hotmail.fr, est Maître de conférences en géographie à l'Université Paris 13 et membre du CRESC (EA 2356). Il a publié récemment :

- Lebeau B., 2014. Economie verte et territoire : un exemple métropolitain. Territoires en mouvement. Article accepté pour publication.

- Lebeau B., 2013. From industrial city to sustainable city: the northern suburbs of Paris yesterday and today. European Spatial Research and Policy, n²-2013, p. 27-40.

- Lebeau B., Vadelorge L, 2012. Enseignement supérieur, recherche et collectivités territoriales. L'Histoire urbaine, $\mathrm{n}^{\circ}$ 33, p. 118-131. 